\title{
A Restricted Version of Wythoff's Game
}

\author{
Wen An Liu* \\ College of Mathematics and Information Science \\ Henan Normal University, Xinxiang, P. R. China \\ liuwenan@126.com \\ Haifeng Li \\ College of Mathematics and Information Science \\ Henan Normal University, Xinxiang, P. R. China \\ flydazhi@sina.com \\ Bei Li \\ College of Mathematics and Information Science \\ Henan Normal University, Xinxiang, P. R. China \\ libeixx1985@126.com
}

Submitted: Jan 5, 2011; Accepted: Sep 20, 2011; Published: Oct 24, 2011

Mathematics Subject Classifications: 91A46, 68R05

\begin{abstract}
E. Duchêne and S. Gravier present the following open problem: In Wythoff's game, each player can either remove at most $R$ tokens from a single heap (i.e. there is an upper bound $R$ on the number of removing tokens), or remove the same number of tokens from both heaps but there is no upper bound on the number of removing tokens. This open problem is investigated and all its P-positions are given.
\end{abstract}

\section{Introduction}

By game we mean a combinatorial game, we restrict our attention to classical impartial games. The winner is the player making the last move. The theory of such games can be found in $[1,2,3]$.

Wythoff's game is played with two heaps of tokens, each player can either remove any number of tokens from a single heap (the Nim rule), or remove the same number of tokens from both heaps (Wythoff's rule).

${ }^{*}$ This work is supported by the National Natural Science Foundation of China under Grant 11171368. 
A position in Wythoff's game is denoted by $(x, y)$, where $x$ denotes the number of tokens in one heap and $y$ denotes the number of tokens in the other heap. The positions from which the previous player can win regardless of the opponent's moves are called $\mathbf{P}$-positions and those from which the next player can win regardless of the opponent's moves are called $\mathbf{N}$-positions.

W.Wythoff ([5]) had given the P-positions of Wythoff's game in 1907. In many papers devoted to variations of Wythoffs game, new rules are adjoined to the original ones. Such variations are called extensions. As an example, in [10] Wythoff's rule is relaxed to take $k>0$ tokens from one heap, $\ell>0$ from the other, subject to $|k-\ell|<s$ where $s>0$ is a fixed integer parameter. Other examples of extensions of Wythoff's game are given in $[7,9,11,12,14]$. There are a few papers where only subsets of Wythoff's moves are allowed (see $[4,8,13])$. Such variations are called restrictions of Wythoff's game. For all these extensions and restrictions of Wythoff's game, the main goal is to find characterizations of the sequence of P-positions, which almost always differs from the original Wythoff's sequence (see [6]).

In page 3605 of [4], the authors discussed one restriction of Wythoff's game, i.e. $R$ radius game: In this game, the length of the moves is bounded by a constant $R \geq 1$. In other words, each player can either remove at most $R$ tokens from a single heap (i.e. there is an upper bound $R$ on the number of removing tokens), or remove the same number of tokens from both heaps and there is an upper bound $R$ on the number of removing tokens. For example, suppose that $R=2$ and a position $(3,7)$, one can only move it to $\{(2,7),(1,7),(3,6),(3,5),(2,6),(1,5)\}$. The authors have given the P-positions of the $R$-radius game.

In the present paper, we consider a restricted version of Wythoff's game. In page 3607 of [4], the authors present the following open problem: In Wythoff's game, each player can either remove at most $R$ tokens from a single heap (i.e. there is an upper bound $R$ on the number of tokens), or remove the same number of tokens from both heaps but there is no upper bound on the number of tokens. For example, suppose that $R=2$ and a position $(3,7)$, one can only move it to $\{(2,7),(1,7),(3,6),(3,5),(2,6),(1,5),(0,4)\}$. For more convenience, we call it "Restricted Move of Wythoff's Game" (RMWG). We give the P-positions of RMWG in this paper.

\section{The P-positions of RMWG}

Definition 1. Let $U \subseteq Z^{\geq 0}=\{k \mid k \geq 0$ is an integer $\}$. By $\operatorname{mex}\{U\}$ we denote the Minimum EXcluded value of $U$, i.e. the smallest nonnegative integer not in $U$. In particular, $\operatorname{mex}\{\emptyset\}=0$.

Each impartial combinatorial game is associated with a digraph $G=(V, E)$, called the game graph. The set $V$ of the vertices is the positions of the game. Given two vertices $v$ and $w$, there is an edge from $v$ to $w$ if there exists a move from the position $v$ to the position $w$.

Definition 2. Suppose that a digraph $G=(V, E)$, a set $S \subseteq V$ is said to be stable if 
there is no edge between any two vertices of $S$. A set $S \subseteq V$ is said to be absorbent if for any $v \in V \backslash S$, there exists $w \in S$ such that $(v, w) \in E$.

Definition 3. Suppose that a digraph $G=(V, E)$, a kernel of $G$ is both stable and an absorbent set of $G$.

The P-positions of a game constitute a kernel of its game graph (see [1]).

The set of the P-positions of Wythoff's game is described in [5]. The symmetry of the game implies that each position $(x, y)$ has its symmetrical $(y, x)$ of the same type, i.e. $(x, y)=(y, x)$. We only discuss the position $(x, y)$ with $x \leq y$.

By $\bigcup_{n=0}^{\infty}\left\{\left(a_{n}, b_{n}\right)\right\}$ we denote the P-positions of Wythoff's game. W. Wythoff $([5])$ had given the formula of $a_{n}$ and $b_{n}$ :

$$
\left\{\begin{array}{l}
a_{n}=\operatorname{mex}\left\{a_{i}, b_{i} \mid 0 \leq i<n\right\} \\
b_{n}=a_{n}+n \\
a_{0}=0, b_{0}=0
\end{array}\right.
$$

The following Table 1 gives a few P-position of Wythoff's game which are determined by Eq. (1).

Table 1: The first few P-positions of Wythoff's game.

\begin{tabular}{c|c|c|c|c|c|c|c|c|c|c|c|c|c|c|c|c|c|c|c|c}
\hline$n$ & 0 & 1 & 2 & 3 & 4 & 5 & 6 & 7 & 8 & 9 & 10 & 11 & 12 & 13 & 14 & 15 & 16 & 17 & 18 & 19 \\
\hline$a_{n}$ & 0 & 1 & 3 & 4 & 6 & 8 & 9 & 11 & 12 & 14 & 16 & 17 & 19 & 21 & 22 & 24 & 25 & 27 & 29 & 30 \\
\hline$b_{n}$ & 0 & 2 & 5 & 7 & 10 & 13 & 15 & 18 & 20 & 23 & 26 & 28 & 31 & 34 & 36 & 39 & 41 & 44 & 47 & 49 \\
\hline
\end{tabular}

Some authors had discussed some properties of $a_{n}$ and $b_{n}$. In order to prove our results, we conclude some new properties in Lemmas 1,2 and 3.

Lemma 1. Let $A=\bigcup_{n=1}^{\infty}\left\{a_{n}\right\}, B=\bigcup_{n=1}^{\infty}\left\{b_{n}\right\}$, where $a_{n}$ and $b_{n}$ are given by Eq. (1). We have the following properties:

(1) $a_{n-1}<a_{n}, b_{n-1}<b_{n}$ for any $n \in Z^{\geq 1}, A \cup B=Z^{\geq 1}, A \bigcap B=\emptyset$.

(2) $a_{n}-a_{n-1} \in\{1,2\}$ and $b_{n}-b_{n-1} \in\{2,3\}$ for any $n \in Z^{\geq 1}$.

(3) If there exist two integers $i, j \in Z^{\geq 1}$ such that $b_{j-1}<a_{i}<b_{j}$, then $i \geq j$ and $a_{i}=i+j-1$.

(4) $a_{a_{n}}=b_{n}-1$ for any $n \in Z^{\geq 1}$.

(5) $a_{b_{n}}=a_{n}+b_{n}$ for any $n \in Z^{\geq 1}$.

(6) $b_{a_{n}}=a_{n}+b_{n}-1$ for any $n \in Z^{\geq 1}$.

(7) $b_{b_{n}}=a_{n}+2 b_{n}$ for any $n \in Z^{\geq 1}$.

Proof. (1) It is obvious by Eq. (1) and the definition of mex.

(2) It is obvious by [14].

(3) It follows from Lemma $1(2)$ that $b_{j}-b_{j-1} \in\{2,3\}$. In this case, there exists an integer $m$ such that $b_{j-1}<m<b_{j}$, i.e. $m \notin B$. By Lemma 1(1), $m \in Z^{\geq 1}=A \bigcup B$ and 
$A \bigcap B=\emptyset$, so $m \in A$, i.e. there exists an integer $i$ such that $b_{j-1}<a_{i}=m<b_{j}$. If $i<j$, then $a_{i}<b_{i} \leq b_{j-1}$, which contradicts $a_{i}>b_{j-1}$, so $i \geq j$.

(case 1) $b_{j}-b_{j-1}=2$. Let $b_{j}=m$, so $b_{j-1}=m-2$. The condition $b_{j-1}<a_{i}<b_{j}$ means that $a_{i}=b_{j}-1=m-1<b_{j}$. By the definition of $a_{i}=\operatorname{mex}\left\{a_{t}, b_{t} \mid 0 \leq t<i\right\}$ and $a_{0}=b_{0}=0$, we have

$$
\{0,1,2, \cdots, m-2\} \subseteq\left\{a_{0}, a_{1}, a_{2}, \cdots, a_{i-1}, b_{1}, b_{2}, \cdots, b_{j-1}, \cdots, b_{i-1}\right\} .
$$

It follows from $b_{j-1}<a_{i}=m-1<b_{j}$ that

$$
\{0,1,2, \cdots, m-2, m-1\} \subseteq\left\{a_{0}, a_{1}, a_{2}, \cdots, a_{i-1}, a_{i}=m-1, b_{1}, b_{2}, \cdots, b_{j-1}\right\} .
$$

On the other hand, it follows from $a_{1}<a_{2}<\cdots<a_{i}=m-1$ and $b_{1}<b_{2}<\cdots<$ $b_{j-1}<a_{i}=m-1$ that

$$
\left\{a_{0}, a_{1}, a_{2}, \cdots, a_{i-1}, a_{i}, b_{1}, b_{2}, \cdots, b_{j-1}\right\} \subseteq\{0,1,2, \cdots, m-2, m-1\} .
$$

Thus we have

$$
\{1,2, \cdots, m-2, m-1\}=\left\{a_{1}, a_{2}, \cdots, a_{i}, b_{1}, b_{2}, \cdots, b_{j-1}\right\} .
$$

Thus $a_{i}=m-1=i+j-1$.

(case 2) $b_{j}-b_{j-1}=3$. Let $b_{j}=m$, so $b_{j-1}=m-3$. The condition $b_{j-1}<a_{i}<b_{j}$ means that $a_{i} \in\{m-1, m-2\}$.

If $a_{i}=m-1=b_{j}-1$, then $a_{i-1}=m-2=a_{i}-1$. We can obtain $\{1,2, \cdots, m-2, m-$ $1\}=\left\{a_{1}, a_{2}, \cdots, a_{i}, b_{1}, b_{2}, \cdots, b_{j-1}\right\}$ and $a_{i}=m-1=i+j-1$ by the same method as (case 1 ).

If $a_{i}=m-2=b_{j}-2$, then $a_{i+1}=m-1=a_{i}+1=b_{j-1}+2$. By the definition of $a_{i}=\operatorname{mex}\left\{a_{t}, b_{t} \mid 0 \leq t<i\right\}$ and $a_{0}=b_{0}=0$, we have

$$
\{1,2, \cdots, m-2\}=\left\{a_{1}, a_{2}, \cdots, a_{i}, b_{1}, b_{2}, \cdots, b_{j-1}\right\} .
$$

Thus $a_{i}=m-2=i+j-1$.

The author of [15] had proved (4), (5), (6) and (7).

Lemma 2. Suppose that an integer $a_{i}$, there exists an unique integer $j \leq i$ such that $b_{j-1}<a_{i}<b_{j}$. For any integer $t$ with $1 \leq t \leq i$, we have

$$
\left\{0,1,2, \cdots, a_{t}-1\right\} \subseteq\left\{a_{1}, a_{2}, \cdots, a_{t-1}, b_{0}, b_{1}, \cdots, b_{j-1}\right\} .
$$

Proof. By the definition of $a_{t-1}=\operatorname{mex}\left\{a_{w}, b_{w} \mid 0 \leq w<t-1\right\}$, we have

$$
\left\{0,1,2, \cdots, a_{t-1}-1\right\} \subseteq\left\{a_{1}, a_{2}, \cdots, a_{t-2}, b_{0}, b_{1}, \cdots, b_{t-2}\right\} .
$$

(Case i) $t \leq j$.

In this case, $t-2<j-1$. It follows from Lemma $1(1)$ that $b_{t-2}<b_{j-1}$. Thus

$$
\left\{0,1,2, \cdots, a_{t-1}-1, a_{t-1}\right\} \subseteq\left\{a_{1}, a_{2}, \cdots, a_{t-2}, a_{t-1}, b_{0}, b_{1}, \cdots, b_{j-1}\right\} .
$$


By Lemma 1(2), $a_{t}-a_{t-1} \in\{1,2\}$. If $a_{t-1}=a_{t}-1$, then Eq. (4) gives Eq. (2); if $a_{t-1}=a_{t}-2$, it implies that $a_{t}-1 \in B$ and $a_{t}-1 \leq a_{j}-1 \leq b_{j-1}$, so Eq. (2) is true.

(Case ii) $t>j$. We distinguish two subcases $t=i$ and $j<t \leq i-1$ :

(Subcase ii.1) $t=i$. By Lemma 1(2), $a_{t}-a_{t-1} \in\{1,2\}$. If $a_{t-1}=a_{t}-1$, then Eq. (4) gives Eq. (2); if $a_{t-1}=a_{t}-2$, we can conclude that $a_{t-1}<b_{j-1}<a_{t}=a_{i}<b_{j}$, i.e. $a_{t}-1=$ $b_{j-1}$. Thus, $\left\{0,1,2, \cdots, a_{t-1}-1, a_{t-1}, a_{t}-1\right\} \subseteq\left\{a_{1}, a_{2}, \cdots, a_{t-2}, a_{t-1}, b_{0}, b_{1}, \cdots, b_{j-1}\right\}$.

(Subcase ii.2) $j<t \leq i-1$. We show that $a_{i-2} \leq b_{j-1}$. In fact, if $a_{i-2}>b_{j-1}$, then $b_{j-1}<a_{i-2}<a_{i-1}<a_{i}<b_{j}$, i.e. $b_{j}-b_{j-1}>3$, which contradicts $b_{j}-b_{j-1} \in\{2,3\}$. We note that $a_{t-1}-1 \leq a_{i-2}-1 \leq b_{j-1}$ for $t \leq i-1$, thus

$$
\left\{0,1,2, \cdots, a_{t-1}-1\right\} \subseteq\left\{a_{1}, a_{2}, \cdots, a_{t-2}, b_{0}, b_{1}, \cdots, b_{j-1}\right\} .
$$

It follows from Eq. (5) that

$$
\left\{0,1,2, \cdots, a_{t-1}-1, a_{t-1}\right\} \subseteq\left\{a_{1}, a_{2}, \cdots, a_{t-2}, a_{t-1}, b_{0}, b_{1}, \cdots, b_{j-1}\right\} .
$$

If $a_{t-1}=a_{t}-1$, then Eq. (6) gives Eq. (2); if $a_{t-1}=a_{t}-2$, then $\left\{0,1,2, \cdots, a_{t-1}-1, a_{t}-2\right\} \subseteq\left\{a_{1}, a_{2}, \cdots, a_{t-2}, a_{t-1}, b_{0}, b_{1}, \cdots, b_{j-1}\right\}$. The condition $a_{t-1}=a_{t}-2$ means that $a_{t}-1 \in B$. It follows from $a_{t}-1<a_{i}\left(a_{t}<a_{i}\right)$ and $b_{j-1}<a_{i}<b_{j}$ that $a_{t}-1 \leq b_{j-1}$, which gives Eq. (2).

Lemma 3. Suppose that an integer $b_{i}=R$ for $i \in Z^{\geq 1}$. For any integer $t$ with $1 \leq t \leq R-i$, we have

$$
\left\{0,1,2, \cdots, a_{t}-1\right\} \subseteq\left\{a_{1}, a_{2}, \cdots, a_{t-1}, b_{0}, b_{1}, \cdots, b_{i}\right\}
$$

Proof. By Lemma 1(4), $a_{a_{i}}=b_{i}-1$, so $a_{R-i}=a_{b_{i}-i}=a_{a_{i}}=b_{i}-1$. By the definition of $a_{t-1}=\operatorname{mex}\left\{a_{w}, b_{w} \mid 0 \leq w<t-1\right\}$, we have

$$
\left\{0,1,2, \cdots, a_{t-1}-1\right\} \subseteq\left\{a_{1}, a_{2}, \cdots, a_{t-2}, b_{0}, b_{1}, \cdots, b_{t-2}\right\} .
$$

(Case i) $1 \leq t \leq i+1$. In this case, $t-2<i$. It follows from Lemma 1(1) that $b_{t-2}<b_{i}$. Thus

$$
\left\{0,1,2, \cdots, a_{t-1}-1, a_{t-1}\right\} \subseteq\left\{a_{1}, a_{2}, \cdots, a_{t-2}, a_{t-1}, b_{0}, b_{1}, \cdots, b_{i}\right\}
$$

By Lemma 1(2), $a_{t}-a_{t-1} \in\{1,2\}$. If $a_{t-1}=a_{t}-1$, then Eq. (9) gives Eq. (7); if $a_{t-1}=a_{t}-2$, then Eq. (9) gives

$$
\left\{0,1,2, \cdots, a_{t-1}-1, a_{t}-2\right\} \subseteq\left\{a_{1}, a_{2}, \cdots, a_{t-2}, a_{t-1}, b_{0}, b_{1}, \cdots, b_{i}\right\}
$$

The condition $a_{t-1}=a_{t}-2$ implies that $a_{t}-1 \in B$. We note that $a_{t}-1 \leq a_{i+1}-1=$ $b_{i+1}-(i+2) \leq b_{i}+3-(i+2) \leq b_{i}$, thus we have Eq. (7).

(Case ii) $i+1<t \leq R-i$. In this case, $a_{t-1}-1<a_{R-i}-1=b_{i}-2<b_{i} \leq b_{t-2}$. Thus

$$
\left\{0,1,2, \cdots, a_{t-1}-1, a_{t-1}\right\} \subseteq\left\{a_{1}, a_{2}, \cdots, a_{t-2}, a_{t-1}, b_{0}, b_{1}, \cdots, b_{i}\right\} .
$$


If $a_{t-1}=a_{t}-1$, then Eq. (10) gives Eq. (7); if $a_{t-1}=a_{t}-2$, then Eq. (10) gives

$$
\left\{0,1,2, \cdots, a_{t-1}-1, a_{t}-2\right\} \subseteq\left\{a_{1}, a_{2}, \cdots, a_{t-2}, a_{t-1}, b_{0}, b_{1}, \cdots, b_{i}\right\} .
$$

The condition $a_{t-1}=a_{t}-2$ means that $a_{t}-1 \in B$. We note that $a_{t}-1 \leq a_{R-i}-1<b_{i}$, thus $\left\{0,1,2, \cdots, a_{t-1}-1, a_{t}-2, a_{t}-1\right\} \subseteq\left\{a_{1}, a_{2}, \cdots, a_{t-2}, a_{t-1}, b_{0}, b_{1}, \cdots, b_{i}\right\}$.

Theorem 4. By $\mathscr{P}$ we denote the set of all P-positions of RMWG. For any integer $R \in Z^{\geq 1}=A \cup B, \mathscr{P}$ can be determined by the following two cases:

(1) If $R=a_{i}$ for an integer $i \in Z^{\geq 1}$, then

$$
\mathscr{P}=\left(\bigcup_{s=0}^{R-i}\left\{\left(a_{s}, b_{s}+(R+1) m\right) \mid m \in Z^{\geq 0}\right\}\right) \bigcup\left(\bigcup_{t=1}^{i}\left\{\left(b_{t}, a_{t}+R+1+(R+1) n\right) \mid n \in Z^{\geq 0}\right\}\right) .
$$

(2) If $R=b_{i}$ for an integer $i \in Z^{\geq 1}$, then

$$
\mathscr{P}=\left(\bigcup_{s=0}^{i}\left\{\left(a_{s}, b_{s}+(R+1) m\right) \mid m \in Z^{\geq 0}\right\}\right) \bigcup\left(\bigcup_{t=1}^{R-i}\left\{\left(b_{t}, a_{t}+R+1+(R+1) n\right) \mid n \in Z^{\geq 0}\right\}\right) \text {. }
$$

Proof. By $r\left(\frac{a}{b}\right)$ we denote the remainder of the division of $a$ by $b$ for $a \geq 0, b>0$. Before we give the proofs of Theorem 4, the following Table 2 lists the first few P-positions, which shows us how to determine the set $\mathscr{P}$ of all P-positions of RMWG game by using

\begin{tabular}{|c|c|c|c|c|c|c|c|c|c|}
\hline$R$ & \multicolumn{9}{|c|}{$\mathscr{P}$} \\
\hline \multirow[t]{2}{*}{$\begin{array}{l}R=1=a_{1} \\
(i=1 \\
R-i=0)\end{array}$} & $s=0$ & $(0,0)$ & $(0,2)$ & $(0,4)$ & $(0,6)$ & $(0,8)$ & $(0,10)$ & & $\left(a_{0}, b_{0}+(R+1) m\right)$ \\
\hline & $t=1$ & $(2,3)$ & $(2,5)$ & $(2,7)$ & $(2,9)$ & $(2,11)$ & $(2,13)$ & $\cdots$ & $\left(b_{1}, a_{1}+(R+1)(n+1)\right)$ \\
\hline \multirow{3}{*}{$\begin{array}{l}R=2=b_{1} \\
(i=1 \\
R-i=1)\end{array}$} & $s=0$ & $(0,0)$ & $(0,3)$ & $(0,6)$ & $(0,9)$ & $(0,12)$ & $(0,15)$ & & $\left(a_{0}, b_{0}+(R+1) m\right)$ \\
\hline & $s=1$ & $(1,2)$ & $(1,5)$ & $(1,8)$ & $(1,11)$ & $(1,14)$ & $(1,17)$ & .. & $\left(a_{1}, b_{1}+(R+1) m\right)$ \\
\hline & $t=1$ & $(2,4)$ & $(2,7)$ & $(2,10)$ & $(2,13)$ & $(2,16)$ & $(2,19)$ & .. & $\left(b_{1}, a_{1}+(R+1)(n+1)\right)$ \\
\hline \multirow{4}{*}{$\begin{array}{l}R=3=a_{2} \\
(i=2, \\
R-i=1)\end{array}$} & $s=0$ & $(0,0)$ & $(0,4)$ & $(0,8)$ & $(0,12)$ & $(0,16)$ & $(0,20)$ & $\cdots$ & $\left(a_{0}, b_{0}+(R+1) m\right)$ \\
\hline & $s=1$ & $(1,2)$ & $(1,6)$ & $(1,10)$ & $(1,14)$ & $(1,18)$ & $(1,22)$ & .. & $\left(a_{1}, b_{1}+(R+1) m\right)$ \\
\hline & $t=1$ & $(2,5)$ & $(2,9)$ & $(2,13)$ & $(2,17)$ & $(2,21)$ & $(2,25)$ & . & $\left(b_{1}, a_{1}+(R+1)(n+1)\right)$ \\
\hline & $t=2$ & $(5,7)$ & $\overline{(5,11)}$ & $(5,15)$ & $(5,19)$ & $(5,23)$ & $(5,27)$ & . & $\left(b_{2}, a_{2}+(R+1)(n+1)\right)$ \\
\hline \multirow{6}{*}{$\begin{array}{l}R=5=b_{2} \\
(i=2 \\
R-i=3)\end{array}$} & $s=0$ & $(0,0)$ & $(0,6)$ & $(0,12)$ & $(0,18)$ & $(0,24)$ & $(0,30)$ & $\cdots$ & $\left(a_{0}, b_{0}+(R+1) m\right)$ \\
\hline & $s=1$ & $(1,2)$ & $(1,8)$ & $(1,14)$ & $(1,20)$ & $(1,26)$ & $(1,32)$ & r. & $\left(a_{1}, b_{1}+(R+1) m\right)$ \\
\hline & $s=2$ & $(3,5)$ & $(3,11)$ & $(3,17)$ & $(3,23)$ & $(3,29)$ & $(3,35)$ & . & $\left(a_{2}, b_{2}+(R+1) m\right)$ \\
\hline & $t=1$ & $(2,7)$ & $\overline{(2,13)}$ & $\overline{(2,19)}$ & $(2,25)$ & $\overline{(2,31)}$ & $\overline{(2,37)}$ & .. & $\left(b_{1}, a_{1}+(R+1)(n+1)\right)$ \\
\hline & $t=2$ & $(5,9)$ & $(5,15)$ & $\overline{(5,21)}$ & $(5,27)$ & $\overline{(5,33)}$ & $\overline{(5,39)}$ & - & $\left(b_{2}, a_{2}+(R+1)(n+1)\right)$ \\
\hline & $t=3$ & $(7,10)$ & $(7,16)$ & $(7,22)$ & $(7,28)$ & $(7,34)$ & $(7,40)$ & & $\left(b_{3}, a_{3}+(R+1)(n+1)\right)$ \\
\hline
\end{tabular}
Theorem 4.

Table 2: The first few P-positions of RMWG game. 
We will show that $\mathscr{P}$ is a stable and absorbent set of the game graph.

Part I: $\mathscr{P}$ is a stable set.

(1) For $R=a_{i}$, there exists a unique integer $j \in Z^{\geq 1}$ such that $b_{j-1}<a_{i}<b_{j}$. By Lemma 1(3), we have $i \geq j$ and $i+j=a_{i}+1=R+1$, i.e. $R-i=j-1$.

The remaining proof is technical. We will find every P-position of RMWG game, then we prove that one can not move it to any P-position of RMWG game. We now consider moves that take from just one heap, we mainly use the fact: let $\left(v_{1}, v_{2}\right)$ and $\left(w_{1}, w_{2}\right)$ be two positions of RMWG, if $v_{1} \neq w_{1}, v_{1} \neq w_{2}, v_{2} \neq w_{1}$ and $v_{2} \neq w_{2}$, then one can not move $\left(v_{1}, v_{2}\right)$ to $\left(w_{1}, w_{2}\right)$ by taking tokens from one heap.

(i) Suppose that $0 \leq s_{1}<s_{2} \leq j-1$, then $a_{s_{1}} \neq a_{s_{2}}, a_{s_{1}} \neq b_{s_{2}}+(R+1) m, b_{s_{1}}+(R+$ 1) $m \neq a_{s_{2}}, b_{s_{1}}+(R+1) m \neq b_{s_{2}}+(R+1) n$. So one can not move $\left(a_{s_{1}}, b_{s_{1}}+(R+1) m\right)$ to $\left(a_{s_{2}}, b_{s_{2}}+(R+1) n\right)$.

Suppose that $0 \leq s \leq j-1,1 \leq t \leq i$. We have $a_{s}<a_{t}+R+1+(R+1) n$, so one can not move $\left(a_{s}, b_{s}+(R+1) m\right)$ to $\left(b_{t}, a_{t}+R+1+(R+1) n\right)$.

(ii) Suppose that $1 \leq t_{1}<t_{0} \leq i$, we now show that if $b_{t_{0}}=a_{t_{1}}+R+1+(R+1) n$ for some $n \in Z^{\geq 0}$, then $n=0$ and $t_{1} \leq j-1$. The inequality $b_{t_{0}} \leq b_{i}=a_{i}+i \leq 2 a_{i}<2(R+1)$ gives $b_{t_{0}}=a_{t_{1}}+R+1$. Suppose $t_{1} \geq j$, then

$$
\begin{aligned}
b_{t_{0}} & \leq b_{i}=b_{i}-R-1+(R+1) \\
& =b_{i}-a_{i}-1+(R+1) \\
& =i-1+R+1=R-j+R+1 \\
& =a_{i}-j+R+1<b_{j}-j+R+1 \\
& =a_{j}+R+1 \leq a_{t_{1}}+R+1,
\end{aligned}
$$

yielding a contradiction. Thus we have: If $b_{t_{0}}=a_{t_{1}}+R+1$, then $t_{1} \leq j-1$. Now suppose $b_{t_{0}}=a_{t_{1}}+R+1$ and one can move $\left(b_{t_{0}}, a_{t_{0}}+R+1\right)$ to $\left(b_{t_{1}}, a_{t_{1}}+R+1\right)$, then

$$
\begin{aligned}
a_{t_{0}}+R+1-b_{t_{1}} & =b_{t_{0}}-t_{0}-a_{t_{1}}-t_{1}+R+1 \\
& =R+1+R+1-\left(t_{0}+t_{1}\right) \\
& \geq R+1+R+1-(i+j-1)=R+2
\end{aligned}
$$

contradicts its bound $R$ (If one can move $\left(b_{t_{0}}, a_{t_{0}}+R+1\right)$ to $\left(b_{t_{1}}, a_{t_{1}}+R+1\right)$, then one must take $a_{t_{0}}+R+1-b_{t_{1}}(\geq R+2)$ tokens from the second heap of $\left(b_{t_{0}}, a_{t_{0}}+R+1\right)$, however, there is an upper bound $R$ when one remove tokens from a single heap, yielding a contradiction).

We also know $b_{t_{0}} \neq b_{t_{1}}, a_{t_{0}}+R+1+(R+1) n \neq a_{t_{1}}+R+1+(R+1) m$, so one can not move $\left(b_{t_{0}}, a_{t_{0}}+R+1+(R+1) n\right)$ to $\left(b_{t_{1}}, a_{t_{1}}+R+1+(R+1) m\right)$.

Suppose that $0 \leq s \leq j-1,1 \leq t \leq i$, one can not move $\left(b_{t}, a_{t}+R+1+(R+1) n\right)$ to $(0,(R+1) m)$ for $b_{t}<a_{t}+R+1$. We consider $1 \leq s \leq j-1$. We know $b_{t} \neq a_{s}$, $a_{t}+R+1+(R+1) n \neq a_{s}, a_{t}+R+1+(R+1) n \neq b_{s}+(R+1) m$. If $1 \leq s=t \leq j-1$, suppose one can move $\left(b_{t}, a_{t}+R+1+(R+1) n\right)$ to $\left(a_{t}, b_{t}\right)$, then $a_{t}+R+1+(R+1) n-a_{t} \geq R+1$, contradict its bound. If $j \leq t \leq i, a_{s} \neq b_{s}+(R+1) m \neq b_{t}, a_{s} \neq b_{s}+(R+1) m \neq$ $a_{t}+R+1+(R+1) n$, so one can not move $\left(b_{t}, a_{t}+R+1+(R+1) n\right)$ to $\left(a_{s}, b_{s}+(R+1) m\right)$. 
We now consider moves that take from both heaps. We mainly use the method: Let $\left(v_{1}, v_{2}\right)$ and $\left(w_{1}, w_{2}\right)$ are two positions of RMWG game. If one can move $\left(v_{1}, v_{2}\right)$ to $\left(w_{1}, w_{2}\right)$ when one take from both heaps, we get $v_{2}-v_{1}=w_{2}-w_{1}$. In order to prove that $\mathscr{P}$ is a stable set, we show that $v_{2}-v_{1} \neq w_{2}-w_{1}$ for $\left(v_{1}, v_{2}\right) \in \mathscr{P}$ and $\left(w_{1}, w_{2}\right) \in \mathscr{P}$. It suffices to show that $r\left(\frac{v_{2}-v_{1}}{R+1}\right) \neq r\left(\frac{w_{2}-w_{1}}{R+1}\right)$. In fact,

$$
\begin{aligned}
& r\left(\frac{b_{s}+(R+1) m-a_{s}}{R+1}\right)=s, 0 \leq s \leq j-1, \\
& r\left(\frac{a_{t}+R+1+(R+1) n-b_{t}}{R+1}\right)=R-t+1,1 \leq t \leq i .
\end{aligned}
$$

If $s=R-t+1$, we have $s+t=R+1$, contradicts $s+t \leq j-1+i=R<R+1$.

(2) $R=b_{i}$. We note that $R-i=b_{i}-i=a_{i} \geq i$ for $i \in Z^{\geq 1}$. We now consider moves that take from just one heap.

(i) Suppose that $0 \leq s_{1}<s_{2} \leq i$, then $a_{s_{1}} \neq a_{s_{2}}, a_{s_{1}} \neq b_{s_{2}}+(R+1) m, b_{s_{1}}+(R+1) m \neq$ $a_{s_{2}}, b_{s_{1}}+(R+1) m \neq b_{s_{2}}+(R+1) n$. So one can not move $\left(a_{s_{1}}, b_{s_{1}}+(R+1) m\right)$ to $\left(a_{s_{2}}, b_{s_{2}}+(R+1) n\right)$.

Suppose that $0 \leq s \leq i, 1 \leq t \leq R-i$. We have $a_{s} \leq a_{i}<b_{i}=R<a_{t}+R+1+(R+1) n$, so one can not move $\left(a_{s}, b_{s}+(R+1) m\right)$ to $\left(b_{t}, a_{t}+R+1+(R+1) n\right)$.

(ii) Suppose that $1 \leq t_{1}<t_{0} \leq R-i$, we now show that if $b_{t_{0}}=a_{t_{1}}+R+1+(R+1) n$ for some $n \in Z^{\geq 0}$, then $n=0$ and $t_{1} \leq i$. The inequality $b_{t_{0}} \leq b_{R-i}=a_{R-i}+R-i=$ $a_{b_{i}-i}+R-i=a_{a_{i}}+R-i=b_{i}-1+R-i<2(R+1)$ gives $b_{t_{0}}=a_{t_{1}}+R+1$. Suppose $t_{1} \geq i+1$, then

$$
\begin{aligned}
b_{t_{0}} & \leq b_{R-i}=a_{R-i}+R-i \\
& =b_{i}-1+R-i \\
& =a_{i}-1+R<a_{t_{1}}+R-1 \\
& <a_{t_{1}}+R+1,
\end{aligned}
$$

yielding a contradiction. Thus we have: If $b_{t_{0}}=a_{t_{1}}+R+1$, then $t_{1} \leq i$. Now suppose $b_{t_{0}}=a_{t_{1}}+R+1$ and one can move $\left(b_{t_{0}}, a_{t_{0}}+R+1\right)$ to $\left(b_{t_{1}}, a_{t_{1}}+R+1\right)$, then

$$
\begin{aligned}
a_{t_{0}}+R+1-b_{t_{1}} & =b_{t_{0}}-t_{0}-a_{t_{1}}-t_{1}+R+1 \\
& =R+1+R+1-\left(t_{0}+t_{1}\right) \\
& \geq R+1+R+1-(R-i+i)=R+2
\end{aligned}
$$

contradicts its bound $R$ (If one can move $\left(b_{t_{0}}, a_{t_{0}}+R+1\right)$ to $\left(b_{t_{1}}, a_{t_{1}}+R+1\right)$, then one must take $a_{t_{0}}+R+1-b_{t_{1}}(\geq R+2)$ tokens from the second heap of $\left(b_{t_{0}}, a_{t_{0}}+R+1\right)$, however, there is an upper bound $R$ when one remove tokens from a single heap, yielding a contradiction).

We also know $b_{t_{0}} \neq b_{t_{1}}, a_{t_{0}}+R+1+(R+1) n \neq a_{t_{1}}+R+1+(R+1) m$, so one can not move $\left(b_{t_{0}}, a_{t_{0}}+R+1+(R+1) n\right)$ to $\left(b_{t_{1}}, a_{t_{1}}+R+1+(R+1) m\right)$.

Suppose that $0 \leq s \leq i, 1 \leq t \leq R-i$, one can not move $\left(b_{t}, a_{t}+R+1+(R+1) n\right)$ to $(0,(R+1) m)$ for $b_{t}=a_{t}+t<a_{t}+R+1$. We consider $1 \leq s \leq i$. We have $a_{1}<a_{2}<\cdots<a_{R-i}=b_{i}-1<R+1$, thus,

$$
\begin{aligned}
& r\left(\frac{a_{t}+R+1+(R+1) n}{R+1}\right)=r\left(\frac{a_{t}}{R+1}\right)=a_{t}, 1 \leq t \leq R-i, \\
& r\left(\frac{b_{s}+(R+1) m}{R+1}\right)=b_{s} \leq b_{i}=R, 0 \leq s \leq i .
\end{aligned}
$$


Then we know $b_{t} \neq a_{s}, a_{t}+R+1+(R+1) n \neq a_{s}, a_{t}+R+1+(R+1) n \neq b_{s}+(R+1) m$. If $1 \leq s=t \leq i$, suppose one can move $\left(b_{t}, a_{t}+R+1+(R+1) n\right)$ to $\left(a_{t}, b_{t}\right)$, then $a_{t}+R+1+(R+1) n-a_{t} \geq R+1$, contradict its bound. If $i+1 \leq t \leq R-i$, $a_{s} \neq b_{s}+(R+1) m \neq b_{t}, a_{s} \neq b_{s}+(R+1) m \neq a_{t}+R+1+(R+1) n$, so one can not move $\left(b_{t}, a_{t}+R+1+(R+1) n\right)$ to $\left(a_{s}, b_{s}+(R+1) m\right)$.

We now consider moves that take from both heaps. The arguments are similar to that of $(1)$.

$$
\begin{aligned}
& r\left(\frac{b_{s}+(R+1) m-a_{s}}{R+1}\right)=s, 0 \leq s \leq i, \\
& r\left(\frac{a_{t}+R+1+(R+1) n-b_{t}}{R+1}\right)=R-t+1,1 \leq t \leq R-i .
\end{aligned}
$$

If $s=R-t+1$, we have $s+t=R+1$, contradicts $s+t \leq i+R-i=R<R+1$. Thus we have proved that $v_{2}-v_{1} \neq w_{2}-w_{1}$ for $\left(v_{1}, v_{2}\right) \in \mathscr{P}$ and $\left(w_{1}, w_{2}\right) \in \mathscr{P}$, i.e. one can not move $\left(v_{1}, v_{2}\right)$ to $\left(w_{1}, w_{2}\right)$ when one take two heaps.

\section{Part II: $\mathscr{P}$ is an absorbent set of the game graph.}

The remaining proof is technical, we mainly use the method: Finding every position except P-positions of RMWG game, then we prove that one can move it to another P-position of RMWG game by moving once.

(1) $R=a_{i}$, then $b_{j-1}<a_{i}<b_{j}$ for $i, j \in Z^{\geq 1}$. Suppose that $\forall(X, Y) \notin \mathscr{P}$, we will prove that one can move $(X, Y)$ to $\left(X^{\prime}, Y^{\prime}\right) \in \mathscr{P}$ by moving once. We only consider $X \leq Y$ and proceed by distinguishing three cases (1.1) $R=a_{1}=1$, (1.2) $R=a_{2}=3$ and (1.3) $R=a_{i}$ for $i>2$ :

(1.1) $R=a_{1}=1$. In this case, $i=j=1$, and $\mathscr{P}=\{(0,2 m),(2,3+2 n)\}$.

(Subcase 1) $X \in\{0,2\}$. If $X=0$, there exists a unique integer $m$ such that $Y=2 m+1$. One can move $(X, Y)$ to $(0,2 m) \in \mathscr{P}$ by taking 1 token from the second heap. If $X=2$, there exists a unique integer $n$ such that $Y=3+2 n+1$. One can move $(X, Y)$ to $(2,3+2 n) \in \mathscr{P}$ by taking 1 token from the second heap; If $X=2$ and $Y=2$, then one can move $(X, Y)=(2,2)$ to $(0,0) \in \mathscr{P}$ by taking 2 tokens from each heap.

(Subcase 2) $X \notin\{0,2\}$. If $X=1$ and $Y=2 n+1$, one can move $(X, Y)$ to $(0,2 n) \in \mathscr{P}$ by taking 1 tokens from each heap. If $X=1$ and $Y=2 n+2$, one can move $(X, Y)$ to $(0,2 n+2) \in \mathscr{P}$ by taking 1 tokens from the first heap.

If $X \geq 3$ and $Y=X+2 n$, one can move $(X, Y)$ to $(0,2 n) \in \mathscr{P}$ by taking $X$ tokens from each heap; If $X \geq 3$ and $Y=X+2 n+1$, one can move $(X, Y)$ to $(2,3+2 n) \in \mathscr{P}$ by taking $X-2$ tokens from each heap.

(1.2) $R=a_{2}=3$. In this case, $i=j=2$. Thus $\mathscr{P}=\{(0,4 m),(1,2+4 m),(2,5+$ $4 n),(5,7+4 n)\}$. The method of this proof is similar to that of (1.1).

(1.3) $R=a_{i}$ for $i>2$. In this case, we have $i>j>2$. In fact, if $i=j$ then $b_{i-1}<a_{i}<b_{j}=b_{i}$, thus $b_{i}-b_{i-1}>b_{i}-a_{i}=i>2$, i.e. $b_{i}-b_{i-1}>3$, which contradicts Lemma 1(2).

We note that

$$
\mathscr{P}=\left(\bigcup_{s=0}^{j-1}\left\{\left(a_{s}, b_{s}+(R+1) m\right) \mid m \in Z^{\geq 0}\right\}\right) \bigcup\left(\bigcup_{t=1}^{i}\left\{\left(b_{t}, a_{t}+R+1+(R+1) n\right) \mid n \in Z^{\geq 0}\right\}\right) .
$$


By Lemma $1(2), b_{i}-b_{i-1} \in\{2,3\}$, i.e. $b_{i}-1 \in A$, thus there exists an integer $k_{0}$ such that $b_{i-1}<a_{k_{0}}=b_{i}-1<b_{i}$. It follows from $a_{k_{0}}>b_{i-1} \geq b_{j}>a_{j}$ that $k_{0}>j$. Let

$$
\begin{aligned}
& A_{1}=\left\{a_{0}, a_{1}, a_{2}, \cdots, a_{j-1}\right\} \\
& A_{2}=\left\{a_{j}, a_{j+1}, \cdots, a_{k_{0}}\right\} \\
& A_{3}=\left\{a_{k_{0}+1}, a_{k_{0}+2}, \cdots\right\} \\
& B_{1}=\left\{b_{1}, b_{2}, b_{3}, \cdots, b_{i}\right\} \\
& B_{2}=\left\{b_{i+1}, b_{i+2}, \cdots\right\} .
\end{aligned}
$$

Thus for any integer $X \in Z^{\geq 0}$, we have $X \in A_{1} \cup A_{2} \cup A_{3} \cup B_{1} \cup B_{2}$. We proceed by discussing two cases $X \in A_{1} \cup B_{1}$ and $X \in A_{2} \cup A_{3} \cup B_{2}$.

(1.3.1) $X \in A_{1} \cup B_{1}$. We distinguish the following four cases: (i) $X=a_{s}$ and $0 \leq s \leq$ $j-1$; (ii) $X=b_{t}$ and $1 \leq t \leq j-1$; (iii) $X=b_{t}$ and $j \leq t \leq i-1$; (iv) $X=b_{i}$.

(i) $X=a_{s}$ for $0 \leq s \leq j-1$.

(i.1) $X \leq Y<b_{s}$. Let $m_{0}=Y-X$, we have $0 \leq m_{0}=Y-X<b_{s}-a_{s}=s \leq j-1$, $X=a_{s}>a_{m_{0}}$ and $Y-\left(X-a_{m_{0}}\right)=Y-X+a_{m_{0}}=m_{0}+a_{m_{0}}=b_{m_{0}}$, thus one can move $(X, Y)$ to $\left(a_{m_{0}}, b_{m_{0}}\right) \in \mathscr{P}$ by taking $X-a_{m_{0}}$ tokens from each heap.

(i.2) $b_{s}+(R+1) m<Y<b_{s}+(R+1) m+R+1$. We note that $0<Y-\left(b_{s}+(R+1) m\right)<$ $R+1$, so one can move $(X, Y)$ to $\left(a_{s}, b_{s}+(R+1) m\right) \in \mathscr{P}$ by taking $Y-\left(b_{s}+(R+1) m\right)$ tokens from the second heap.

(ii) $X=b_{t}$ for $1 \leq t \leq j-1$.

(ii.1) $X \leq Y<a_{t}+R+1$. We note that $0<X-a_{t} \leq Y-a_{t}<a_{t}+R+1-a_{t}=R+1$, so one can move $(X, Y)$ to $\left(b_{t}, a_{t}\right)=\left(a_{t}, b_{t}\right) \in \mathscr{P}$ by taking $Y-a_{t}$ tokens from the second heap.

(ii.2) $a_{t}+R+1+(R+1) n<Y<a_{t}+R+1+(R+1) n+R+1$. We note that $0<$ $Y-\left(a_{t}+R+1+(R+1) n\right)<R+1$, so one can move $(X, Y)$ to $\left(b_{t}, a_{t}+R+1+(R+1) n\right) \in \mathscr{P}$ by taking $Y-\left(a_{t}+R+1+(R+1) n\right)$ tokens from the second heap.

(iii) $X=b_{t}$ for $j \leq t \leq i-1$. We distinguish the following three subcases:

(iii.1) $X \leq Y \leq b_{t}+j-1=a_{t}+t+R-i$. Let $m_{0}=Y-X$, we have $0 \leq m_{0}=$ $Y-X \leq j-1, X=b_{t}>b_{j-1}>a_{j-1} \geq a_{m_{0}}$ and $Y-\left(X-a_{m_{0}}\right)=Y-X+a_{m_{0}}=b_{m_{0}}$, so one can move $(X, Y)$ to $\left(a_{m_{0}}, b_{m_{0}}\right) \in \mathscr{P}$ by taking $X-a_{m_{0}}$ tokens from each heap.

(iii.2) $a_{t}+t+R-i+1=b_{t}+j \leq Y<a_{t}+R+1$.

We now prove that one can move $(X, Y)$ to $\left(X^{\prime}, Y^{\prime}\right) \in \mathscr{P}$ by taking tokens from the first heap. Let $s_{0}=\left(a_{t}+t+R+1\right)-Y$, we have $j \leq t<s_{0} \leq i$ and

$$
r\left(\frac{Y}{R+1}\right)=r\left(\frac{a_{t}+t+R-s_{0}+1}{R+1}\right)=a_{t}+t-s_{0}<a_{t}<a_{i}=R .
$$

It follows from Lemma 2 that $r\left(\frac{Y}{R+1}\right) \in\left\{a_{1}, a_{2}, \cdots, a_{t-2}, a_{t-1}, b_{0}, b_{1}, \cdots, b_{j-1}\right\}$.

If there exists $s \in\{0,1,2, \cdots, j-1\}$ such that $r\left(\frac{Y}{R+1}\right)=b_{s}=a_{t}+t-s_{0}$, then we have $Y=b_{s}+R+1$ by $b_{s} \leq b_{j-1}<b_{j}+j \leq b_{t}+j \leq Y<a_{t}+R+1<2(R+1)$. Thus

$$
0<X-a_{s}=b_{t}-\left(b_{s}-s\right)=b_{t}-b_{t}+s_{0}+s \leq i+j-1=R<R+1,
$$

one can move $(X, Y)$ to $\left(a_{s}, b_{s}+R+1\right) \in \mathscr{P}$ by taking $X-a_{s}$ tokens from the first heap. 
If there exists $q \in\{1,2, \cdots, t-1\}$ such that $r\left(\frac{Y}{R+1}\right)=a_{q}=a_{t}+t-s_{0}$, then we have $Y=a_{q}+R+1$ by $a_{q}<a_{t}<b_{t}+j \leq Y<a_{t}+R+1<2(R+1)$. Thus

$$
0<X-b_{q}=b_{t}-a_{q}-q=b_{t}-b_{t}+s_{0}-q<s_{0} \leq i \leq a_{i}=R
$$

so one can move $(X, Y)$ to $\left(b_{q}, a_{q}+R+1\right) \in \mathscr{P}$ by taking $X-b_{q}$ tokens from the first heap.

(iii.3) $a_{t}+R+1+(R+1) n<Y<a_{t}+R+1+(R+1) n+R+1$. In this case, $0<$ $Y-\left(a_{t}+R+1+(R+1) n\right)<R+1$, so one can move $(X, Y)$ to $\left(b_{t}, a_{t}+R+1+(R+1) n\right) \in \mathscr{P}$ by taking $Y-\left(a_{t}+R+1+(R+1) n\right)$ tokens from the second heap.

(iv) $X=b_{i}$. We distinguish the following two subcases:

(iv.1) $X \leq Y<a_{i}+R+1=b_{i}+R+1-i$. Let $m_{0}=Y-X$, it follows from $0 \leq Y-X=Y-b_{i}<R+1-i=j$ that $0 \leq m_{0}=Y-X \leq j-1$. We note that $X=b_{i}>b_{j}>a_{j-1} \geq a_{m_{0}}$ and $Y-\left(X-a_{m_{0}}\right)=m_{0}+a_{m_{0}}=b_{m_{0}}$, one can move $(X, Y)$ to $\left(a_{m_{0}}, b_{m_{0}}\right) \in \mathscr{P}$ by taking $X-a_{m_{0}}$ tokens from each heap.

(iv.2) $a_{i}+R+1+(R+1) n<Y<a_{i}+R+1+(R+1) n+(R+1)$. We have $0<$ $Y-\left(a_{i}+R+1+(R+1) n\right)<R+1$, so one can move $(X, Y)$ to $\left(b_{i}, a_{i}+R+1+(R+1) n\right) \in \mathscr{P}$ by taking $Y-\left(a_{i}+R+1+(R+1) n\right)$ tokens from the second heap.

(1.3.2) $X \in A_{2} \cup A_{3} \cup B_{2}$.

(i) $X \in A_{2}$. In this case, $X=a_{k}$ for $j-1<k \leq k_{0}$, and there exists an integer $\ell$ such that $b_{\ell-1}<a_{k}<b_{\ell}$. We can conclude that $1 \leq \ell \leq i$. In fact, if $\ell>i$ then $b_{\ell-1} \geq b_{i}>a_{k_{0}} \geq a_{k}$, which contradicts $b_{\ell-1}<a_{k}$. For any $Y \geq X=a_{k},(X, Y) \notin \mathscr{P}$, there exists an integer $m \in Z^{\geq 0}$ such that $a_{k}+(R+1) m \leq Y<a_{k}+R+1+(R+1) m$. We distinguish the following three subcases:

(i.1) $a_{k}+(R+1) m \leq Y \leq a_{k}+j-1+(R+1) m$. Let $m_{0}=Y-(X+(R+1) m)$, we have $0 \leq m_{0} \leq j-1<k, X=a_{k}>a_{m_{0}}$ and $Y-\left(X-a_{m_{0}}\right)=m_{0}+(R+1) m+a_{m_{0}}=$ $b_{m_{0}}+(R+1) m$, thus one can move $(X, Y)$ to $\left(a_{m_{0}}, b_{m_{0}}+(R+1) m\right)$ by taking $X-a_{m_{0}}$ tokens from each heap.

(i.2) $a_{k}+R-i+1+(R+1) m=a_{k}+j+(R+1) m \leq Y \leq a_{k}+R-\ell+1+(R+1) m$.

We now prove that one can move $(X, Y)$ to $\left(X^{\prime}, Y^{\prime}\right) \in \mathscr{P}$ by taking tokens from the first heap. Let $p=\left(a_{k}+R+1+(R+1) m\right)-Y$, we have $\ell \leq p \leq i$ and

$$
\begin{aligned}
r\left(\frac{Y}{R+1}\right) & =r\left(\frac{a_{k}+R+1+(R+1) m-p}{R+1}\right) \\
& =a_{k}-p<b_{\ell}-\ell \\
& =a_{\ell} \leq a_{i}=R .
\end{aligned}
$$

We note that $1 \leq \ell \leq i$ and $b_{j-1}<a_{i}<b_{j}$, it follows from Lemma 2 that $\left\{0,1,2, \cdots, a_{\ell}-1\right\} \subseteq\left\{a_{1}, a_{2}, \cdots, a_{\ell-1}, b_{0}, b_{1}, \cdots, b_{j-1}\right\}$. Thus,

$$
r\left(\frac{Y}{R+1}\right) \in\left\{a_{1}, a_{2}, \cdots, a_{\ell-2}, a_{\ell-1}, b_{0}, b_{1}, \cdots, b_{j-1}\right\} .
$$

If there exists $s \in\{0,1,2, \cdots, j-1\}$ such that $r\left(\frac{Y}{R+1}\right)=b_{s}=a_{k}-p$, then we have

$$
\begin{aligned}
Y & \geq a_{k}+j+(R+1) m \\
& >a_{j-1}+j-1+(R+1) m \\
& =b_{j-1}+(R+1) m \\
& \geq b_{s}+(R+1) m,
\end{aligned}
$$


and

$$
\begin{aligned}
Y & \leq a_{k}+R-\ell+1+(R+1) m \\
& <b_{\ell}-\ell+R+1+(R+1) m \\
& =a_{\ell}+R+1+(R+1) m \\
& \leq a_{i}+R+1+(R+1) m \\
& <R+1+R+1+(R+1) m .
\end{aligned}
$$

The Eqs. (11) and (12) imply that $Y=b_{s}+R+1+(R+1) m$ and

$$
0<X-a_{s}=X-\left(b_{s}-s\right)=X-a_{k}+p+s=p+s \leq i+j-1=R<R+1,
$$

hence one can move $(X, Y)$ to $\left(a_{s}, b_{s}+R+1+(R+1) m\right) \in \mathscr{P}$ by taking $X-a_{s}$ tokens from the first heap.

If there exists $q \in\{1,2, \cdots, \ell-1\}$ such that $r\left(\frac{Y}{R+1}\right)=a_{q}=a_{k}-p$, we have

$$
a_{q}<a_{\ell} \leq a_{i}<b_{j}=a_{j}+j \leq a_{k}+j .
$$

Thus $a_{q}+(R+1) m<a_{k}+j+(R+1) m \leq Y$ and Eq. (12) imply that $Y=a_{q}+R+1+$ $(R+1) m$. We note that

$$
0<X-b_{q}=X-\left(a_{q}+q\right)=X-\left(a_{k}-p+q\right)=p-q<p \leq i \leq a_{i}=R<R+1,
$$

so one can move $(X, Y)$ to $\left(b_{q}, a_{q}+R+1+(R+1) m\right) \in \mathscr{P}$ by taking $X-b_{q}$ tokens from the first heap.

(i.3) $a_{k}+R-\ell+1+(R+1) m<Y<a_{k}+R+1+(R+1) m$. Let $m_{0}=Y-(X+(R+1) m)$, we have $R-\ell+1<m_{0}<R+1$. We note that

$$
X=a_{k}>b_{\ell-1}=b_{R+1-(R+1-(\ell-1))} \geq b_{R+1-m_{0}},
$$

and

$$
\begin{aligned}
Y-\left(X-b_{R+1-m_{0}}\right) & =(Y-X)+b_{R+1-m_{0}} \\
& =\left(m_{0}+(R+1) m\right)+\left(R+1-m_{0}+a_{R+1-m_{0}}\right) \\
& =a_{R+1-m_{0}}+R+1+(R+1) m,
\end{aligned}
$$

so one can move $(X, Y)$ to $\left(b_{R+1-m_{0}}, a_{R+1-m_{0}}+R+1+(R+1) m\right) \in \mathscr{P}$ by taking $X-b_{R+1-m_{0}}$ tokens from each heap.

(ii) $X \in A_{3} \cup B_{2}$. We can conclude that $X>b_{i}$. In fact, if $X \in B_{2}$ then $X \geq b_{i+1}>b_{i}$; if $X \in A_{3}$ then $X \geq a_{k_{0}+1} \geq a_{k_{0}}+1=b_{i}$, on the other hand, $X \in A_{3} \subseteq A$, so $X \neq b_{i}$, i.e. $X>b_{i}$.

Suppose that $Y \geq X$ and let $v=r\left(\frac{Y-X}{R+1}\right) \in\{0,1,2, \cdots, R\}$, i.e. there exists an integer $n$ such that $Y-X=(R+1) n+v$. If $0 \leq v \leq j-1$, then $X>b_{i}>a_{i}>a_{j}>a_{v}$ and

$$
Y-\left(X-a_{v}\right)=Y-X+a_{v}=b_{v}+(R+1) n,
$$

so one can move $(X, Y)$ to $\left(a_{v}, b_{v}+(R+1) n\right) \in \mathscr{P}$ by taking $X-a_{v}$ tokens from each heap. If $j \leq v \leq R$, then $X>b_{i}=b_{R+1-j} \geq b_{R+1-v}$ and

$$
\begin{aligned}
Y-\left(X-b_{R+1-v}\right) & =Y-X+b_{R+1-v} \\
& =v+(R+1) n+a_{R+1-v}+R+1-v \\
& =a_{R+1-v}+R+1+(R+1) n,
\end{aligned}
$$


so one can move $(X, Y)$ to $\left(b_{R+1-v}, a_{R+1-v}+R+1+(R+1) n\right) \in \mathscr{P}$ by taking $X-b_{R+1-v}$ tokens from each heap.

(2) $R=b_{i}$ for $i \in Z^{\geq 1}$. Given $\forall(X, Y) \notin \mathscr{P}$, we will prove that one can move $(X, Y)$ to $\left(X^{\prime}, Y^{\prime}\right) \in \mathscr{P}$ by moving once. We only consider $X \leq Y$ and proceed by distinguishing two cases (2.1) $R=b_{1}=2$ and (2.2) $R=b_{i}$ for $i>1$ :

(2.1) $R=b_{1}=2$. In this case, $R-i=i=1$, and $\mathscr{P}=\{(0,3 m),(1,2+3 m),(2,4+3 n)\}$.

(Subcase 1) $X \in\{0,1,2\}$. If $X=0$ and $Y=3 m+k, k \in\{1,2\}$, one can move $(X, Y)$ to $(0,3 m) \in \mathscr{P}$ by taking $k(\leq R)$ tokens from the second heap.

If $X=1$ and $Y=3 m$, one can move $(X, Y)$ to $(0,3 m) \in \mathscr{P}$ by taking 1 token from the first heap; if $X=1$ and $Y=3 m+1$, one can move $(X, Y)$ to $(0,3 m) \in \mathscr{P}$ by taking 1 token from the each heap.

If $X=2$ and $Y=3 m$, one can move $(X, Y)$ to $(0,3 m) \in \mathscr{P}$ by taking 2 tokens from the first heap. If $X=2$ and $Y=3 m+2$, then one can move $(X, Y)$ to $(0,3 m) \in \mathscr{P}$ by taking 2 tokens from each heap; if $X=2$ and $Y=3$, then one can move $(X, Y)$ to $(1,2) \in \mathscr{P}$ by taking 1 token from each heap.

(Subcase 2) $X \notin\{0,1,2\}$. We have $X \geq 3$. If $Y=X+3 m$, one can move $(X, Y)$ to $(0,3 m) \in \mathscr{P}$ by taking $X$ tokens from each heap; if $Y=X+3 m+1$, one can move $(X, Y)$ to $(1,2+3 m) \in \mathscr{P}$ by taking $X-1$ tokens from each heap; if $Y=X+3 m+2$, one can move $(X, Y)$ to $(2,4+3 m) \in \mathscr{P}$ by taking $X-2$ tokens from each heap.

(2.2) $R=b_{i}$ for $i \geq 2$. In this case, we have $R-i=b_{i}-i=a_{i}>i$.

We note that

$$
\mathscr{P}=\left(\bigcup_{s=0}^{i}\left\{\left(a_{s}, b_{s}+(R+1) m\right) \mid m \in Z^{\geq 0}\right\}\right) \bigcup\left(\bigcup_{t=1}^{R-i}\left\{\left(b_{t}, a_{t}+R+1+(R+1) n\right) \mid n \in Z^{\geq 0}\right\}\right) .
$$

By Lemma $1(2), b_{R-i}-b_{R-i-1} \in\{2,3\}$, i.e. $b_{R-i}-1 \in A$, thus there exists an integer $k_{0}$ such that $b_{R-i-1}<a_{k_{0}}=b_{R-i}-1<b_{R-i}$. It follows from $a_{k_{0}}=b_{R-i}-1 \geq b_{i+1}-1=$ $a_{i+1}+i+1-1>a_{i+1}$ that $k_{0}>i+1$. Let

$$
\begin{aligned}
& A_{1}=\left\{a_{0}, a_{1}, a_{2}, \cdots, a_{i}\right\} \\
& A_{2}=\left\{a_{i+1}, a_{i+2}, \cdots, a_{k_{0}}\right\} \\
& A_{3}=\left\{a_{k_{0}+1}, a_{k_{0}+2}, \cdots\right\}, \\
& B_{1}=\left\{b_{1}, b_{2}, b_{3}, \cdots, b_{R-i}\right\} \\
& B_{2}=\left\{b_{R-i+1}, b_{R-i+2}, \cdots\right\} .
\end{aligned}
$$

Thus for any integer $X \in Z^{\geq 0}$, we have $X \in A_{1} \cup A_{2} \cup A_{3} \cup B_{1} \cup B_{2}$. We proceed by discussing two cases (2.2.1) $X \in A_{1} \cup B_{1}$ and (2.2.2) $X \in A_{2} \cup A_{3} \cup B_{2}$ :

(2.2.1) $X \in A_{1} \cup B_{1}$. We distinguish the following four subcases: (i) $X=a_{s}$ and $0 \leq s \leq i$; (ii) $X=b_{t}$ and $1 \leq t \leq i$; (iii) $X=b_{t}$ and $i+1 \leq t \leq R-i-1$; (iv) $X=b_{R-i}$.

(i) $X=a_{s}$ for $0 \leq s \leq i$.

(i.1) $X \leq Y<b_{s}$. Let $m_{0}=Y-X$, we have $0 \leq m_{0}=Y-X<b_{s}-a_{s}=s \leq i$, $X=a_{s}>a_{m_{0}}$ and $Y-\left(X-a_{m_{0}}\right)=Y-X+a_{m_{0}}=m_{0}+a_{m_{0}}=b_{m_{0}}$, thus one can move $(X, Y)$ to $\left(a_{m_{0}}, b_{m_{0}}\right) \in \mathscr{P}$ by taking $X-a_{m_{0}}$ tokens from each heap. 
(i.2) $b_{s}+(R+1) m<Y<b_{s}+(R+1) m+R+1$. We note that $0<Y-\left(b_{s}+(R+1) m\right)<$ $R+1$, so one can move $(X, Y)$ to $\left(a_{s}, b_{s}+(R+1) m\right) \in \mathscr{P}$ by taking $Y-\left(b_{s}+(R+1) m\right)$ tokens from the second heap.

(ii) $X=b_{t}$ for $1 \leq t \leq i$.

(ii.1) $X \leq Y<a_{t}+R+1$. We note that $0<X-a_{t} \leq Y-a_{t}<a_{t}+R+1-a_{t}=R+1$, so one can move $(X, Y)$ to $\left(b_{t}, a_{t}\right)=\left(a_{t}, b_{t}\right) \in \mathscr{P}$ by taking $Y-a_{t}$ tokens from the second heap.

(ii.2) $a_{t}+R+1+(R+1) n<Y<a_{t}+R+1+(R+1) n+R+1$. We note that $0<$ $Y-\left(a_{t}+R+1+(R+1) n\right)<R+1$, so one can move $(X, Y)$ to $\left(b_{t}, a_{t}+R+1+(R+1) n\right) \in \mathscr{P}$ by taking $Y-\left(a_{t}+R+1+(R+1) n\right)$ tokens from the second heap.

(iii) $X=b_{t}$ for $i+1 \leq t \leq R-i-1$. We distinguish the following three subcases:

(iii.1) $X \leq Y \leq b_{t}+i$. Let $m_{0}=Y-X$, we have $0 \leq m_{0}=Y-X \leq i, X=b_{t}>$ $b_{i}>a_{i} \geq a_{m_{0}}$ and $Y-\left(X-a_{m_{0}}\right)=Y-X+a_{m_{0}}=b_{m_{0}}$, so one can move $(X, Y)$ to $\left(a_{m_{0}}, b_{m_{0}}\right) \in \mathscr{P}$ by taking $X-a_{m_{0}}$ tokens from each heap.

(iii.2) $b_{t}+R+1-(R-i)=b_{t}+i+1 \leq Y<a_{t}+R+1=b_{t}+R+1-t$.

We now prove that one can move $(X, Y)$ to $\left(X^{\prime}, Y^{\prime}\right) \in \mathscr{P}$ by taking tokens from the first heap. Let $s_{0}=\left(b_{t}+R+1\right)-Y$, we have $t<s_{0} \leq R-i$ and

$$
r\left(\frac{Y}{R+1}\right)=r\left(\frac{b_{t}+R+1-s_{0}}{R+1}\right)=b_{t}-s_{0}=a_{t}+t-s_{0}<a_{t}<a_{R-i}=b_{i}-1<b_{i}=R .
$$

It follows from Lemma 3 that

$$
r\left(\frac{Y}{R+1}\right) \in\left\{0,1,2, \cdots, a_{t}-1\right\} \subseteq\left\{a_{1}, a_{2}, \cdots, a_{t-2}, a_{t-1}, b_{0}, b_{1}, \cdots, b_{i}\right\} .
$$

If there exists $s \in\{0,1,2, \cdots, i\}$ such that $r\left(\frac{Y}{R+1}\right)=b_{s}=b_{t}-s_{0}$, then we have $Y=b_{s}+R+1$ by $b_{s} \leq b_{i}<b_{i}+i<b_{t}+i<Y<a_{t}+R+1<a_{R-i}+R+1=$ $b_{i}-1+R+1<2(R+1)$. Thus

$$
0<X-a_{s}=b_{t}-\left(b_{s}-s\right)=b_{t}-b_{t}+s_{0}+s \leq R-i+i=R<R+1,
$$

one can move $(X, Y)$ to $\left(a_{s}, b_{s}+R+1\right) \in \mathscr{P}$ by taking $X-a_{s}$ tokens from the first heap.

If there exists $q \in\{1,2, \cdots, t-1\}$ such that $r\left(\frac{Y}{R+1}\right)=a_{q}=b_{t}-s_{0}$, then we have $Y=a_{q}+R+1$ by $a_{q}<a_{t}<b_{t}+i<Y<a_{t}+R+1<2(R+1)$. Thus

$$
0<X-b_{q}=b_{t}-a_{q}-q=b_{t}-b_{t}+s_{0}-q<s_{0} \leq R-i<R,
$$

so one can move $(X, Y)$ to $\left(b_{q}, a_{q}+R+1\right) \in \mathscr{P}$ by taking $X-b_{q}$ tokens from the first heap.

(iii.3) $a_{t}+R+1+(R+1) n<Y<a_{t}+R+1+(R+1) n+R+1$. In this case, $0<$ $Y-\left(a_{t}+R+1+(R+1) n\right)<R+1$, so one can move $(X, Y)$ to $\left(b_{t}, a_{t}+R+1+(R+1) n\right) \in \mathscr{P}$ by taking $Y-\left(a_{t}+R+1+(R+1) n\right)$ tokens from the second heap.

(iv) $X=b_{R-i}$. We distinguish the following two subcases:

(iv.1) $X \leq Y<a_{R-i}+R+1$. Let $m_{0}=Y-X$, It follows from $0 \leq Y-X=$ $Y-b_{R-i}<a_{R-i}+R+1-\left(a_{R-i}+R-i\right)=i+1$ that $0 \leq m_{0}=Y-X \leq i$. We note that 
$X=b_{R-i}>b_{i}>a_{i} \geq a_{m_{0}}$ and $Y-\left(X-a_{m_{0}}\right)=m_{0}+a_{m_{0}}=b_{m_{0}}$, one can move $(X, Y)$ to $\left(a_{m_{0}}, b_{m_{0}}\right) \in \mathscr{P}$ by taking $X-a_{m_{0}}$ tokens from each heap.

(iv.2) $a_{R-i}+R+1+(R+1) n<Y<a_{R-i}+R+1+(R+1) n+(R+1)$. We have $0<Y-\left(a_{R-i}+R+1+(R+1) n\right)<R+1$, so one can move $(X, Y)$ to $\left(b_{R-i}, a_{R-i}+R+\right.$ $1+(R+1) n) \in \mathscr{P}$ by taking $Y-\left(a_{R-i}+R+1+(R+1) n\right)$ tokens from the second heap.

(2.2.2) $X \in A_{2} \bigcup A_{3} \bigcup B_{2}$.

(i) $X \in A_{2}$. In this case, $X=a_{k}$ for $i<k \leq k_{0}$, and there exists an integer $\ell$ such that $b_{\ell-1}<a_{k}<b_{\ell}$. We can conclude that $1 \leq \ell \leq R-i$. In fact, if $\ell>R-i$, i.e. $\ell-1 \geq R-i$, then $b_{\ell-1}>b_{\ell-1}-1 \geq b_{R-i}-1=a_{k_{0}} \geq a_{k}$, which contradicts with $b_{\ell-1}<a_{k}$. For any $Y \geq X=a_{k},(X, Y) \notin \mathscr{P}$, there exists an integer $m \in Z^{\geq 0}$ such that $a_{k}+(R+1) m \leq Y<a_{k}+R+1+(R+1) m$. We distinguish the following three subcases:

(i.1) $a_{k}+(R+1) m \leq Y \leq a_{k}+i+(R+1) m$. Let $m_{0}=Y-(X+(R+1) m)$, we have $0 \leq m_{0} \leq i<k, X=a_{k}>a_{m_{0}}$ and $Y-\left(X-a_{m_{0}}\right)=m_{0}+(R+1) m+a_{m_{0}}=b_{m_{0}}+(R+1) m$, thus one can move $(X, Y)$ to $\left(a_{m_{0}}, b_{m_{0}}+(R+1) m\right)$ by taking $X-a_{m_{0}}$ tokens from each heap.

(i.2) $a_{k}+R+1-(R-i)+(R+1) m=a_{k}+i+1+(R+1) m \leq Y \leq a_{k}+R+1-\ell+(R+1) m$.

We now prove that one can move $(X, Y)$ to $\left(X^{\prime}, Y^{\prime}\right) \in \mathscr{P}$ by taking tokens from the first heap. Let $p=\left(a_{k}+R+1+(R+1) m\right)-Y$, we have $\ell \leq p \leq R-i$ and

$$
\begin{aligned}
r\left(\frac{Y}{R+1}\right) & =r\left(\frac{a_{k}+R+1+(R+1) m-p}{R+1}\right) \\
& =a_{k}-p<b_{\ell}-\ell \\
& =a_{\ell} \leq a_{R-i} \\
& =b_{i}-1<R .
\end{aligned}
$$

It follows from Lemma 3 that

$$
r\left(\frac{Y}{R+1}\right) \in\left\{0,1,2, \cdots, a_{\ell}-1\right\} \subseteq\left\{a_{1}, a_{2}, \cdots, a_{\ell-1}, b_{0}, b_{1}, \cdots, b_{i}\right\}
$$

If there exists $s \in\{0,1,2, \cdots, i\}$ such that $r\left(\frac{Y}{R+1}\right)=b_{s}=a_{k}-p$, then we have

$$
\begin{aligned}
Y & \geq a_{k}+i+1+(R+1) m \\
& >a_{i}+i+(R+1) m \\
& =b_{i}+(R+1) m \\
& \geq b_{s}+(R+1) m,
\end{aligned}
$$

and

$$
\begin{aligned}
Y & \leq a_{k}+R-\ell+1+(R+1) m \\
& <b_{\ell}-\ell+R+1+(R+1) m \\
& =a_{\ell}+R+1+(R+1) m \\
& \leq a_{R-i}+R+1+(R+1) m \\
& <R+1+R+1+(R+1) m .
\end{aligned}
$$

The Eqs. (15) and (16) imply that $Y=b_{s}+R+1+(R+1) m$. We note that

$$
0<X-a_{s}=X-\left(b_{s}-s\right)=X-a_{k}+p+s=p+s \leq R-i+i=R<R+1,
$$


hence one can move $(X, Y)$ to $\left(a_{s}, b_{s}+R+1+(R+1) m\right) \in \mathscr{P}$ by taking $X-a_{s}$ tokens from the first heap.

If there exists $q \in\{1,2, \cdots, \ell-1\}$ such that $r\left(\frac{Y}{R+1}\right)=a_{q}=a_{k}-p$, we have

$$
a_{q}<a_{\ell} \leq a_{R-i}<b_{i}=a_{i}+i<a_{k}+i+1 .
$$

Thus $a_{q}+(R+1) m<a_{k}+i+1+(R+1) m \leq Y$ and Eq. (16) imply that $Y=$ $a_{q}+R+1+(R+1) m$. We note that

$$
0<X-b_{q}=X-\left(a_{q}+q\right)=X-\left(a_{k}-p+q\right)=p-q<p \leq R-i<R<R+1,
$$

so one can move $(X, Y)$ to $\left(b_{q}, a_{q}+R+1+(R+1) m\right) \in \mathscr{P}$ by taking $X-b_{q}$ tokens from the first heap.

(i.3) $a_{k}+R-\ell+1+(R+1) m<Y<a_{k}+R+1+(R+1) m$. Let $m_{0}=Y-(X+(R+1) m)$, we have $R-\ell+1<m_{0}<R+1$. We note that

$$
X=a_{k}>b_{\ell-1}=b_{R+1-(R+1-(\ell-1))} \geq b_{R+1-m_{0}},
$$

and

$$
\begin{aligned}
Y-\left(X-b_{R+1-m_{0}}\right) & =(Y-X)+b_{R+1-m_{0}} \\
& =\left(m_{0}+(R+1) m\right)+\left(R+1-m_{0}+a_{R+1-m_{0}}\right) \\
& =a_{R+1-m_{0}}+R+1+(R+1) m,
\end{aligned}
$$

so one can move $(X, Y)$ to $\left(b_{R+1-m_{0}}, a_{R+1-m_{0}}+R+1+(R+1) m\right) \in \mathscr{P}$ by taking $X-b_{R+1-m_{0}}$ tokens from each heap.

(ii) $X \in A_{3} \cup B_{2}$. We can conclude that $X>b_{R-i}$. In fact, if $X \in B_{2}$ then $X \geq$ $b_{R-i+1}>b_{R-i}$; if $X \in A_{3}$ then $X \geq a_{k_{0}+1} \geq a_{k_{0}}+1=b_{R-i}$, on the other hand, $X \in A_{3} \subseteq$ $A$, so $X \neq b_{R-i}$, i.e. $X>b_{R-i}$.

Suppose that $Y \geq X, v=r\left(\frac{Y-X}{R+1}\right) \in\{0,1,2, \cdots, R\}$, i.e. there exists an integer $n$ such that $Y-X=(R+1) n+v$. If $0 \leq v \leq i$, then $X>b_{R-i}>a_{R-i}>a_{i} \geq a_{v}$ and

$$
Y-\left(X-a_{v}\right)=Y-X+a_{v}=b_{v}+(R+1) n,
$$

so one can move $(X, Y)$ to $\left(a_{v}, b_{v}+(R+1) n\right) \in \mathscr{P}$ by taking $X-a_{v}$ tokens from each heap. If $i+1 \leq v \leq R$, then $X>b_{R-i} \geq b_{R+1-v}$ and

$$
\begin{aligned}
Y-\left(X-b_{R+1-v}\right) & =Y-X+b_{R+1-v} \\
& =v+(R+1) n+a_{R+1-v}+R+1-v \\
& =a_{R+1-v}+R+1+(R+1) n,
\end{aligned}
$$

so one can move $(X, Y)$ to $\left(b_{R+1-v}, a_{R+1-v}+R+1+(R+1) n\right) \in \mathscr{P}$ by taking $X-b_{R+1-v}$ tokens from each heap.

\section{Acknowledgements}

The authors are grateful to the responsible editor and the anonymous referees for their valuable comments and suggestions, which have greatly improved the earlier version of this paper. The research is supported by the National Natural Science Foundation of China under Grant 11171368. 


\section{References}

[1] E.R. Berlekamp, J.H. Conway, and R.K. Guy, Winning Ways for Your Mathematical Plays, Academic Press, 1982.

[2] C.L. Bouton, Nim, a game with a complete mathematical theory, Ann. Math. 3 (1905), 35-39.

[3] J.H. Conway, On Numbers and Games, Academic Press, London, 1976.

[4] E. Duchêne, and S. Gravier, Geometrical extensions of Wythoff's game, Disc. Math. 309 (2009), 3595-3608.

[5] W. Wythoff, A modification of the game of Nim, Nieuw Arch. Wisk. 7 (1907), 199202.

[6] E. Duchêne, A.S. Fraenkel, R.J. Nowakowski, and M. Rigo, Extensions and restrictions of Wythoff's game preserving its P-positions, Journal of Combinatorial Theory, Series A 117 (2010), 545-567.

[7] I.G. Connell, A generalization of Wythoff's game, Canad. Math. Bull. 2 (1959), 181190.

[8] E. Duchêne, A.S. Fraenkel, S. Gravier, and R.J. Nowakowski, Another bridge between Nim and Wythoff, Australasian J. of Combinatorics 44 (2009), 43-56.

[9] A.S. Fraenkel, Euclid and Wythoff games, Discrete Math. 304 (2005), 65-68.

[10] A.S. Fraenkel, How to beat your Wythoff games' opponent on three fronts, Amer. Math. Monthly 89 (1982), 353-361.

[11] A.S. Fraenkel, Heap games, Numeration systems and Sequences, Annals of Combinatorics 2 (1998), 197-210.

[12] A.S. Fraenkel, and E. Reisner, The game of End-Wythoff, Games of No Chance III, Proc. BIRS Workshop on Combinatorial Games, July 2005, Banff, Alberta, Canada, MSRI Publ., M.H. Albert and R.J. Nowakowski, Eds., Vol. 56, Cambridge University Press, Cambridge, (2009) 329-347 .

[13] A.S. Fraenkel, and M. Lorberbom, Nimhoff games, J. Combin. Theory (Ser. A) 58 (1991), 1-25.

[14] Xinyu Sun, and D. Zeilberger, On Fraenkel's N-heap Wythoff's conjectures, Annals of Combinatorics 8 (2004), 225-238.

[15] C. Kimberling, Complementary equations and Wythoff sequences, Journal of Integer Sequences, Vol. 11 (2008), Article 08.3.3. 